\title{
Adults Are Not Big Children: What Brain Magnetic Resonance Imaging Findings \\ Tell Us About Differences in Pediatric and Adult Cerebral Malaria
}

\author{
2020 \\ Chandy C. John \\ Ryan White Center for Pediatric Infectious Diseases and Global Health, Indiana University School of \\ Medicine, Indianapolis, Indiana, USA
}

Historically, research studies of infections in children have lagged behind those in adults. There are many reasons for this, including the smaller population of children, resulting in smaller numbers for research studies; a reluctance to subject children to the procedures required and the potential harms from new interventions; and a lack of financial incentive with the smaller market for drugs for pediatric conditions. However, the lack of pediatric data has led to suboptimal understanding and treatment of some infectious diseases that are seen less often in children than adults. The phrase "children are not little adults" has been a mantra for pediatricians who advocate for the need for research studies in children, because the pathogenesis and course of a specific disease in children is often very different from that seen in adults. The converse is also true, although less remarked on: adults are not big children (well, most are not). For diseases that occur more often in children, such as cerebral malaria (CM), an understanding of differences in pathogenesis and complications in adults versus children is critical to designing the best interventions for each population.

Magnetic resonance imaging (MRI) is a particularly useful method of assessing disease pathogenesis in CM. Pioneering case series of CM in adults in Thailand [1] and children in Malawi [2] led to a landmark

This is the author's manuscript of the work published in final edited form as:

John, C. C. (2020). Adults Are Not Big Children: What Brain Magnetic Resonance Imaging Findings Tell Us About Differences in Pediatric and Adult Cerebral Malaria. Clinical Infectious Diseases, ciaa1659. https://doi.org/10.1093/cid/ciaa1659 
study in Malawi in which brain swelling was shown to be associated with death in children with CM [3]. Fewer studies have been done in adults with $\mathrm{CM}$, as $\mathrm{CM}$ occurs far less often in adults, and few radiographic predictors of mortality in adults with $\mathrm{CM}$ have been documented.

Cerebral malaria is unusual in that it presents in different age groups based on transmission intensity of an area: in the areas of highest transmission, it is uncommon; in areas of moderate transmission, it presents in young children; and in areas of very low transmission, it typically presents in adolescents and young adults. For this reason, most studies of $\mathrm{CM}$ from a single area consist of exclusively pediatric or adult populations. In this issue of Clinical Infectious Diseases, Sahu and colleagues report findings of a study of CM in Rourkela, India, an area in which both children and adults present with $\mathrm{CM}$. This unusual circumstance provided them the opportunity to compare MRI findings in children and adults with CM at the same site and compare fatal with nonfatal $\mathrm{CM}$ in both groups. By evaluating not only brain volume but also the apparent diffusion coefficient (ADC), which decreases with cytotoxic edema and increases with vasogenic edema, they were able to define the type of brain edema occurring adults and children. They show that brain swelling increased with age, that children and adults had different localization of restricted diffusion, and that fatal $\mathrm{CM}$ was associated with decreased ADC in adults, suggesting cytotoxic edema, whereas in children, 2 of the 3 children who died had high ADC values, consistent with vasogenic edema, and one had brainstem herniation and low ADC values. In addition, levels of lipocalin-2, a marker of global brain hypoxia, were higher in adults than children, and highest in adults with fatal CM, suggesting that global brain hypoxia may be a more common phenomenon in adults than children, and may be a factor in mortality in adults. Overall, the findings provide new evidence that adults with CM are more likely to have global hypoxia and cytotoxic edema, both of which are associated with death in adults, while in children, vasogenic edema and brain swelling are more common and more likely to lead to death. 
Studies like the study by Sahu and colleagues require persistence, patience, and funding support that goes beyond the usual 3-5 years provided by standard grants. Enrollment and study completion for this study took 6 years, as the number of cases each year that met study criteria was relatively small and malaria generally occurred within a 4-month season each year. Without long-term support and infrastructure, the study would not have enrolled sufficient study participants to evaluate the MRI differences between adult and pediatric CM. Studies like this also require substantial dedicated infrastructure on site-for example, the 1.5-T Siemens Symphony MRI scanner that was immediately available for very sick patients, along with dedicated clinical and radiology expertise to provide the needed clinical care and to conduct and interpret the MRI testing. Many areas in which CM is most common lack the resources to support and maintain this level of sophisticated care and imaging. Visionary groups like those in Malawi, Thailand, and India have performed a great service in getting high-quality MRI machines to their sites, supporting the infrastructure and personnel required to maintain them, and obtaining funding for the high-quality studies they have conducted. Together, these studies have given us complementary new insights into the pathogenesis of CM in adults and children, insights we hope will lead to successful adjunctive therapy for pediatric and adult CM.

So what adjunctive interventions might we use for adult or pediatric CM? The authors point out that the presence of cytotoxic, rather than vasogenic, edema in adults may explain the failure of mannitol treatment for adults with CM [4]. They also point to a recent study that showed effectiveness of a glutamate antagonist in reducing cytotoxic edema and reversing CM pathology in a murine model [5]. Unfortunately, we need still more data on what happens in pediatric and adult CM to better guide therapies. With the small number of deaths in children $(n=3)$ and adults $(n=4)$ in this study, it is difficult to say what role that cytotoxic edema might play, in addition to vasogenic edema, in leading to death in children. In addition, there are differences in African and Indian pediatric populations with CM that could affect disease pathogenesis in the 2 populations. The pediatric cohort in the study by Sahu et 
al was older than most African cohorts (the mean age in this cohort was 7.8 years for nonfatal $\mathrm{CM}$ and 5 years for CM, whereas the mean age was a little above 4 years for the Malawi MRI cohort [3]). More strikingly, CM in both adults and children in the cohort recruited by Sahu et al occurred more frequently in males than females ( $78 \%$ of children with $\mathrm{CM}$ and $72 \%$ of adults with $\mathrm{CM}$ were male), whereas the Malawi cohort had an approximately equivalent proportion of males and females (51\% males) [3]). Whether this represents a sex-based risk of CM or differences in health-seeking behavior is not known, but it deserves further study. Furthermore, one might assume that mannitol would provide better results in children with $\mathrm{CM}$, who have more vasogenic edema than adults with $\mathrm{CM}$. However, mannitol also did not improve outcomes in pediatric $\mathrm{CM}[6]$. So, there is more to learn before we embark on randomized clinical trials of interventions in children and adults with $\mathrm{CM}$.

Studies like the study by Sahu and colleagues point us in the right direction to get there. As evidence accumulates from well-conducted studies by this group and others, we will get closer to adjunctive interventions that can successfully reduce mortality in $\mathrm{CM}$ in adults and children. The study by Sahu et al provides good reason to believe the interventions in adults and children may not be the same, showing once again that children are not small adults, and also that adults are not big children. 


\section{References}

1. Looareesuwan S, Wilairatana $\mathrm{P}$, Krishna S, et al. Magnetic resonance imaging of the brain in patients with cerebral malaria. Clin Infect Dis 1995; 21:300-9.

2. Potchen MJ, Kampondeni SD, Seydel KB, et al. Acute brain MRI findings in 120 Malawian children with cerebral malaria: new insights into an ancient disease. AJNR Am J Neuroradiol 2012; $33: 1740-6$.

3. Seydel KB, Kampondeni SD, Valim C, et al. Brain swelling and death in children with cerebral malaria. N Engl J Med 2015; 372:1126-37.

4. Mohanty S, Mishra SK, Patnaik R, et al. Brain swelling and mannitol therapy in adult cerebral malaria: a randomized trial. Clin Infect Dis 2011; 53:349-55.

5. Riggle BA, Sinharay S, Schreiber-Stainthorp W, et al. MRI demonstrates glutamine antagonistmediated reversal of cerebral malaria pathology in mice. Proc Natl Acad Sci USA 2018; 115:E12024-33.

6. Namutangula B, Ndeezi G, Byarugaba JS, Tumwine JK. Mannitol as adjunct therapy for childhood cerebral malaria in Uganda: a randomized clinical trial. Malar J 2007; 6:138. 